II. Aus dem städtischen Krankenhause Moabit in Berlin. (Abth. des Herrn Geh. Med.-Rath Prof. Dr. Renvers.)

\section{Zur Kenntniss der myelogenen Leukämie.} Von Dr. Hans Hirsehfeld und Dr. Ernst Tobjas, ehemaligen Assistenzärzten.

So zahlreich auch die Fälle von myelogener Leukämie sind, die bisher in der Litteratur beschrieben wurden, so sind sie doch zum grössten Theil so lückenhaft bezüglich des Blutbefundes beobachtet, dass eine wissenschaftliche Verwerthung nur weniger derselben möglich ist.

Wir wollen deshalb in den folgenden Zeilen zwei sehr gellau klinisch und pathologisch-allatonisch untersuchte Fälle dieser Erkrankung beschreiben, welche im Krankenhause Moabit auf der Abtheilung des Herrn Geh.-Rath Renvers beobachtet worden sind und in mancher Hinsicht recht interessante Befunde erheben liessen.

Bei dem einen Patienten verfügen wir nur über klinische Daten, da er nach längerem Aufenthalt das Krankenhaus und zugleich Berlin verliess; bei dem andern Leukämiker, der im Krankenhause starb, konnte eine eingehende makroskopische und mikroskopische pathologisch-anatomische Untersucliung ausgeführt werden.

Auch die Angaben Löwit's über die parasitäre Natur der Leukämie und die Möglichkeit ihrer experimentellen Uebertragung haben wir nachprïfen können.

Wir theilen zunächst die Krankengeschichte unseres ersten Falles mit.

Fall 1. Anton Roszycki, 19 Jahre alt, Gärtner: Aufgenommen am 27. November 1899. Eltern und vier Geschwister leben und sind gesund. Vor zwei Tagen starkes Nasenbluten. Seitdem Herzklopfen und aufgetriebener Leib. Infektion und Potus werden geleugnet.

Status praesens: Untermittelgrosser, schwächlich gebanter Mann. Puls voll und leicht beschleunigt. Kein Fieber. Herzorenzen normal; über allen Ostien systolische Geräısche. Lungen ohne Abweichung.

Die Untersuchung des Abdomens ergiebt das Bestehen eines grossen Milztumors. Derselbe erstreckt sich bis etwa drei Finger breit unter den linken Rippenbogen. Er ist hart und lässt sich von allen Seiten gut abgrenzen. Die Palpation desselben ist nicht schmerzhaft. Urin ohne Eiweiss und Zucker.

Die Knochen sind auf Belklopfen nicht schmerzhaft. Die füllbaren Lymphdrüsen nicht geschwollen.

Die Untersuchung eines frischen Blnttropfens ergiebt das Vorhandensein einer enormen Menge von weissen Blutkörperchen. Hämoglobingehalt $35 \%$. Zahl der Erythrocyten 1.157500. Zahl der weissen 500000 .

Krankheitsverlauf: Wiederholt starkes Nasenbluten. Die Milz wäohst langsam. Kein Fieber. Am 24. April 1900, als Patient auf seinen Wunsch entlassen wurde, war die Milz noch einmal so gross, als im Anfang. Sonst bietet der Krankheitsverlauf nichts bemerkenswerthes.

Aus der nachstehenden Tabelle geht hervor, dass das Prozentverhältniss der verschiedenen Leukocytenformen ausserordentlich grossen Schwankungen im Verlauf der Krankheit ausgesetzt war. Am constantesten blieb die relative Zahl der mehrkernigen neutroplilien Zellen. Die grossen und kleinen einkernigen Zellen, die Mast- und die eosinophilen Zellen boten das wechselndste Zahlenverhältniss dar. Die Mastzellen sowie die grossen und kleinen

1) Von einer "Conservirung“ des Fleisches durch schwefligsanres Natron kann übrigens nicht die Rede sein. Wohl bleibt durch den Sulfitzusatz die rothe Farbe des Fleisches, namentlich des Hackfleisches lange Zeit erhalten; indessen findet, wie nenere exakte Untersuchungen von Gürtner, Stroscher, Meyer ergeben haben, im derartig behandelten Fleisch keinerlei Abtödtung von Keimen statt, sondern es kommt höchstens eine vorübergehende, kaum nennenswerthe Entwicklungshemmung zu Stande. Mit schwefligsaurem Salz versetztes Hackfleisch sieht nach vielen Stunden noch lebhaft roth aus, erweckt also durch seine Farbe den Anschein frisch zerkleinerten Fleisches, während sich thatsächlich die in ihm enthaltenen Keime in grosser Mcnge entwickelt haben. einkernigen Leukocyten fehlten an einzelnen Tagen völlig. Unter den Myelocyten fiel der schon mehrfach beschriebene, ausserordentlich verschiedene Gehalt der einzelnen Zellen an Granulis auf. Diejenigen Granula, welche nur in geringer Menge im Protoplasma zerstreut lagen, hatten in Triacid- wie in MethylenblauEosinfärbung einen deutlich blauen Farbenton, ein Zeiclien ihrer Jugendlichkeit, während die reichlich vorhandenen rothviolett in Triacidpräparaten gefärbt waren. Auch unter dell mehrkernigen Leukocyten sah man vielfach solche mit Granulis, die einen deutlich blauen Farbenton im Triacid angenommen hatten. Die eben besprochenen Farbenunterschiede der Granula waren noch deutlicher ansgesprochen in den eosinophilen Zellen. Neben solchen mit gleichmässig gesättigt rothen Körnuugen, sah man solche, die theils rothe, theils blaue Granula fiihrten, ferner solche, die ausschliesslich Granula mit tief blaurothem Farbenton enthielten. Letztere waren bedeutend voluminösel als die gewöhnlichen eosinophilen Granula.

\begin{tabular}{ll|c|c|c|c|c|c}
\multicolumn{7}{c}{ B lutb ef un d. } \\
\hline \hline
\end{tabular}

Die Affinität junger Granula zu den basischen Farbstoffen hat Hirschfeld ${ }^{1}$ ) zuerst für die eosinophilen Granula gelegentlich von Untersuchungen über die Histogenese der Knochenmarkzellen festgestellt. Auch Ehrlich und Lazarus geben in ihrer "Anämie" an, dass junge Granula eine basophile Quote enthielten. Vor kurzem ist dem einen von uns $^{2}$ ) der Nachweis gelungen, dass die neutrophilen Granula im Jugendzustande sich sowohl mit neutralen wie mit basischen Farben tingiren. Wie sich in dieser Beziehung die neutrophilen Granula bei der Leukämie verhalten, konnten wir nicht feststellen, da die vorliegende Arbeit früher abgeschlossen war, als der eben genannte Befund gemacht wurde. ${ }^{3}$ )

Fall 2. Arbeiter Paul Neunherz, 31 Jahre alt, aufgenommen am 8. Mai 1899 .

Anamnese: Bis Ende Januar stets gesund. Keine hereditäre Belastung. Seit Ende Januar Anschwellıng der Füsse, seit einiger, nicht genau zu bestimmender Zeit ständig zunehmende Anschwellıng des Leibes.

Status praesens: Mässig kräftig gebauter Mann. Normale Herzgrenzen mit ziemlich lauten systolischen Geräuschen. Brustorgane ohne Besonderheiten. Temperatur 37,5 ${ }^{\circ}$. Leib stark aufgetrieben. Die Milz reicht bis zur Inguinalgegend, in der Mitte bis zum Nabel herab, so dass fast die Hälfte des Abdomens von ihr ausgefüllt wird. Oberfläche glatt, nicht schmerzhaft. Kein Ascites. Leichtes Oedem der Beine in der Malleolengegend. Urin leicht getrübt. Mikroskopisch negativer Befund. Schwere Veränderungen im Augenhintergrund. Rechts zahlreiche kleine Blutungen, Papillitis; links dasselbe. Nur fällt hier besondérs eine starke Schlängelıng der Venen auf. Blut: myelogene Leukämie. Der Befund wird im Zusammenhang genaı erörtert werden. 16. Mai. 424000 Leukocyten.

1. Juni. Kein Fieber mehr.

1) Hirschfeld, Zur Histogenese der granulirten Knochenmarkzellen. Virchow's Archiv Bd. CLIII

${ }^{2}$ ) Hirschfeld, Ueber Veränderungen der multinukleären Leıkocyten bei Infektionskrankheiten. Berliner klinische Wochenschrift 1901 No. 29.

3) Inzwischen ist es mir gelungen, in einem neuerdings beobachteten Fall von Leukämie festzustellen, dass sich die Granula fast sämmtlicher Myelocyten und einzelner polynukleären Elemente ausser mit Triacid auch mit wässriger Methylenblaulösung färbten. (Anmerkung bei der Correktur:) 
27. Juni. Die Temperatur ist auf $39,6^{\circ}$ angestiegen. Lautes Reiben über der noch grösser gewordenen Milz

15. Juli. Fieber und Reiben verschwunden. Sonst status idem.

20. September. Hämoglobin 40\%. Leukocyten 228 300, Erythrocyten 3150000 , also $14: 1$.

3. Oktober. Hämoglobin 40\%. Leukocyten 300000 . Zeitweilig leichte Temperaturanstiege mit Schmerzen in der Milzgegend and Reibegeräuschen daselbst.

9. Dezember. Periostitis des linken Unterschenkels. Erneute Temperatursteigerung mit starken Schmerzen.

12. Dezember. Starke Anschwellung des linken Fussgelenks.

23. Dezember. Rückgang beider Schwellungen. Patient ist fieber-

frei. Leukocyten 300000 , Erythrocyten 3000000 , also $10: 1$.

6. Januar 1900 . Leukocyten 327000 .

17. Januar. Leukocyten 372500 .

21. Januar. Leukocyten 331250.

28. Januar. Anschwellung der Halsdrüsen. Hämoglobin 40\%. Leukocyten 403333 .

3. Februar. Patient hat seit zwei Wochen sehr schwankende Temperaturen, zwischen 37 und $39^{\circ}$. Die Untersuchung ergiebt einen profusen Katarrh beider Lungen ohne Dämpfung. Leukocyten 110937, Erythrocyten 850000

15. Februar. Katarıh hält an mit hohem Fieber. Dazu stellt sich eine Periostitis rechts und eine Schwellung der Innenseite des rechten Fussgelenks ein.

27. Februar. Schwellung und Fieber lassen nach. Letzteres hält sich auf $38^{\circ}$. Anhaltender Katarrh der Lungen.

30. März. Patient hat im ganzen 19 Pfund an Gewicht verloren.

4. April. Der Katarrh bessert sich. Kein Fieber mehr.

2. Mai. Am linken $\mathrm{Ohr}$ hat sich, auf dem Helixknorpel aufsitzend, ein kleiner walnussgrosser Tumor gebildet, mit granulirender Oberfläche. Als er schnell wächst, wird er entfernt. Beschreibung folgt.

3. Juni. Katarrhalische Pneumonie des rechten Unterlappens mit hohem Fieber.

19. Juni. Katarrh gebessert. Nur noch leichte abendliche Temperatursteigerungen.

27. Juni. Rezidivirende Pneumonie, die bis zum 4. Juli anhält und einen erneuten Gewichtsverlust von 5 Pfund zur Folge hat.

10. August. Hämoglobin $45 \%$ Leukocyten 283333 , Erythrocyten 1900000 . Der diffuse Katarrh weicht nicht mehr. Anhaltende hohe Temperatursteigerungen. Zunehmende Kachexie.

15. September. Exitus letalis.

Sektionprotokoll (Professor Langerhans; Obduzent Dr. Graupner): Die Lungen sind etwas derb, nur in den vorderen Abschnitten weicher. Im linken Oberlappen ist ein von schiefrigem Gewebe eingeschlossener käsiger Heerd. In beiden Lungen sind zahlreiche submiliare grauweisse Knötchen zerstreut. In der linken Pleura vereinzelte Knötchen.

Milz $32 \mathrm{~cm}$ lang, $17 \mathrm{~cm}$ breit, $9 \mathrm{~cm}$ dick. Die Kapsel ist zum grössten Theil kallös verdickt, sehr derb, fast hart; das graurothe Milzparenchym ist in der Schnittfläche überall durchsetzt von dunkelrothen, streifigen Zügen, die das erstere in einzelne Abschnitte sondern. Von der stärksten kallösen Verdickung gehen gelbe bis gelbweisse gallertartige Zïge, theils durch das Milzparenchym hindurch, theils nur oberflächlich hinein. An dem oberen Pol ein etwa walnussgrosser Knoten von derselben Beschaffenheit mitten im Gewebe.

\section{Blutbefund.}

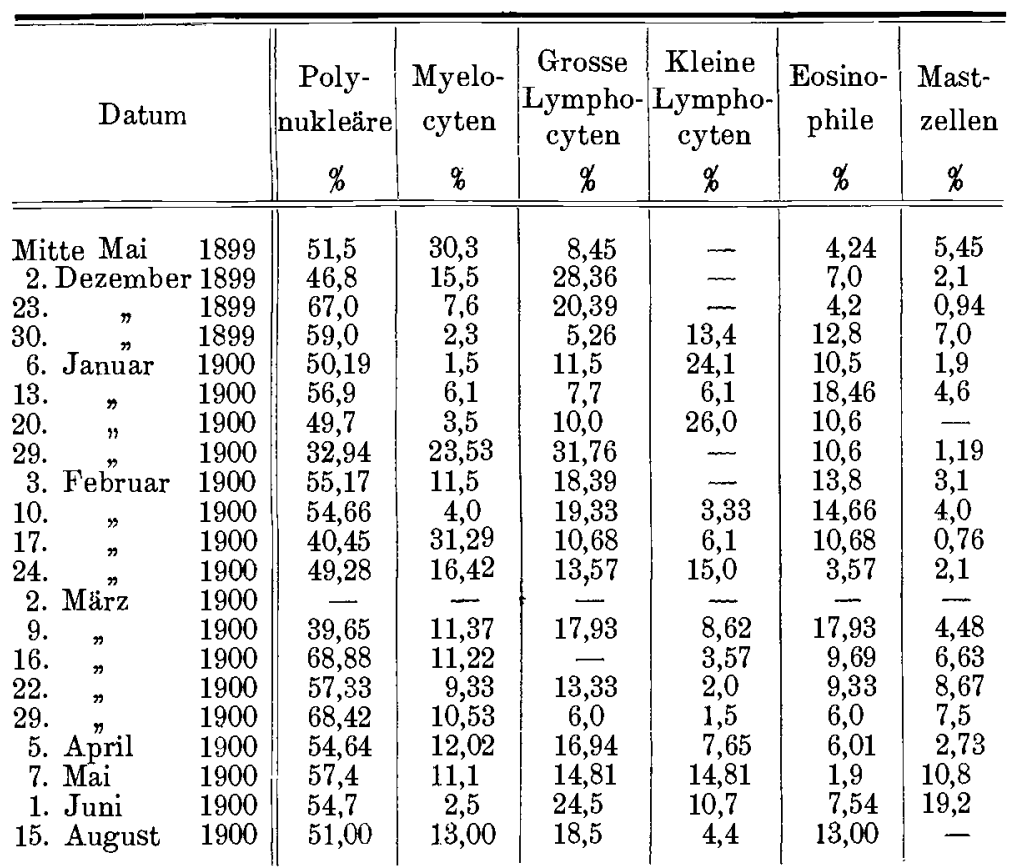

Die mesenterialen Lymphdrüsen sind bis Taubeneigrösse geschwollen. An der Oberfläche der Leber tuberkelähnliche Gebilde.

A us der Sektionsdiagnose: Leukaemia lienalis. Tuberculosis miliaris pulmonum (?), pleurarum, hepatis. Induratio pigmentosa et tubercula obsoleta apicis utriusque pulmonis. Lymphoma multiplex lienis, hepatis, renis dextri. In den langen Röhrenknochen fehlt Fettmark fast gänzlich. Das Knochenmark bildet eine weisse, von einzelnen rothen Partieen durchsetzte Masse von fast weicher Consistenz.

Auch bei diesem Patienten sind die erheblichen Abweichungen, welche das Blutbild der verschiedenen Tage zeigt, sehr bemerkenswerth.

Um die Einzelheiten des Blutbefundes zu schildern, geben wir einen kurzen Auszug aus unseren bezüglichen Notizen.

Mitte Mai 1899. 1. Rothe Blutkörperchen: geringe Grössenunterschiede, keine Poikilocytose, vereinzelte Normoblasten, wenig poly chromatophile Elemente, weniğ Blutplättchen. 2. Weisse Blutzellen: Ein sehr grosser Theil der Leukocyten erscheint einkernig und von abnormer Grösse mit neutrophilen Granulationen (Myelocyten), 4,24\% eosinophile Zellen, darunter einzelne abnorm gross und mit einem runden Kern: einige dieser Zellen enthalten blaue und rothe Granula (Triacid). Eosinophile und neutrophile Zwergformen, 5,45\% Mastzellen.

2. Dezember 1899. Die Zahl der nicht granulirten grossen Lymphocyten hat bedeutend zugenommen $(28,36 \%)$, desgleichen die Zahl der eosinophilen ( $7 \%$ ), die der Mastzellen hat abgenommen.

23. Dezember. Bedeutende Abnahme der Myelocyten- und Mast. zellen.

30. Dezember. Auch die grossen Lymphocyten haben abgenommen, plötzlich 13,4\% kleine Lymphocyten, die bisher ganz fehlten. Auch Zunahme der eosinophilen und Mastzellen.

In ähnlicher Weise verhielten sich die Zahlen weiter wechselnd. Im ganzen zeigten die geringsten Schwankungen die polynukleären neutrophilen Elemente. Kleine Lymphocyten und Mast. zellen wurden an einzelnen Tagen völlig vermisst.

Wir wollen nun noch eine genauere Beschreibung der einzelnen Leukocytenformen geben, die in diesem Falle eine viel grössere Polymorphie zeigten als im ersten.

An den mehrkernigen Zellen fielen erhebliche Grössenunterschiede auf; neben Riesenformen fanden wir Zwergformen, sowohl neutrophile wie eosinophile. Eine geringe Menge der mehrkernigen Zellen hatte ein völlig granulafreies Protoplasma. Solche Zellen hat bisher nur Arneth ${ }^{1}$ ) beschrieben, und Hirschfeld ${ }^{2}$ ) sah sie in einem Falle von Knochenmarksarkomatose. In einem von Ehrlich beschriebenen Falle von posthämorrhagischer Anämie fehlten in allen mehrkernigen Zellen die Granula. Zap. pert und Blachstein ${ }^{3}$ ) beschrieben bei Leukämie Myelocyten mit granulafreiem Protoplasma. Man muss mit Ehrlich und Lazarus annehmen, dass unter Umständen die Fähigkeit gewisser Zellen, neutrophile Substanzen zu bilden, erlischt.

Die Farbenunterschiede der neutrophilen und eosinophilen Granula verhielten sich hier genau so wie in dem zuerst beschriebenen Falle.

Eine eigenthümliche Zellform waren ganz kleine polymorphoder mehrkernige Zellen, die sich von den Zwergformen dadurch unterschieden, dass sie nur ganz wenige Granula enthielten, die tiefroth bis blauviolett gefärbt waren.

Einige dieser Zellen enthielten stark lichtbrechende, ungefärbte Körnchen und waren somit als Mastzellen aufzufassen; andere enthielten neben letzteren die oben beschriebenen Granula. Dass Mastzellen ausser den eigentlichen Mastzellengranulis auch solche mit anderer Farbenaffinität enthalten, ist von Arnold und von Engel behauptet, von Ehrlich aber bestritten worden. Unsere Befunde sprechen für die Richtigkeit der Angaben Arnold's und Engel's. Wahrscheinlich verhalten sich eben die einzelnen Fälle von Leukämie vel'schieden. Die Mastzellen zeigten eine starke Polymorphie; neben den gewöhnlichen Formen, kleinen ein- oder mehrkernigen Elementen, fanden wir auch voluminöse Zellen vom Habitus der grossen Lymphocyten mit zweifellosen Mastzellenkörnungen

Aus dem vorstehenden Sektionsprotokoll geht hervor, dass ausser der Leukämie noch eine Tuberkulose bestand, die im Leben

1) Arneth, Hämatologischer Befund zu W. v. Leube: Ueber einen Fall von rapide verlaufender schwerer Anämie mit gleichzeitig leukämischer Beschaffenheit des Blutes. Archiv für klinische Medizin Bd. LXIX.

2) Hirschfeld, Ueber Blutbefunde bei Knochenmarkstumoren Fortschritte der Medizin 1901, No. 29.

3) Zappert, Blachstein, sh. Ehrlich-Lazarus: Die Anämie Theil I, S. 126 . 
nicht so charakteristische Erscheinungen gemacht hatte, dass sie hätte diagnostizirt werden können.

Der Ausgangspunkt dieser Tuberkulose waren wohl alte Heerde in den Spitzen (Induratio pigmentosa et tuberculosa obsoleta apicum pulmonum). Offenbar hat die Leukämie die neue Entwickelung und Propagation der alten Tuberkulose begünstigt. Es handelte sich um eine Miliartuberkulose. Die Lunge und die Pleura waren übersät von Tuberkeln, auch in der Leber waren einige sichtbar. Die mikroskopische Untersuchung bestätigte die makroskopische Diagnose und zeigte, dass auch kleine käsige Heerde in der Milz und in den Lymphdrüsen bestanden, wo man sie mit blossem Auge nicht sehen konnte. Alle miliaren Tuberkel enthielten Riesenzellen, Tuberkelbazillen konnten wir im Schnitt nicht nachweisen.

Die Combination von Leukämie und Tuberkulose ist recht selten.

Virchow (Virchow's Archiv Bd. II, S. 587) theilt einen Fall von Leukämie mit, die wir nach der Beschreibung jetzt als myelogene bezeichnen würden, in welchem, ausgehend von einer alten Bronchialdrüsentuberkulose, frische Tuberkel der Pleura und der Lungen entstanden waren.

Bamberger (Schmidt's Jahrbücher Bd. XCVII, S. 204) beschreibt einen Fall von lymphatischer Leukämie, in welchem zugleich eine alte kavernöse Phthise bestand.

Honnsell (Schmidt's Jahrbücher Bd. CXXII, S. 47) sah ebenfalls die Combination von Leukämie und Phthise.

Francksen (Inaugural-Dissertation, Göttingen 1892) beobachtete einen Fall von Leukämie, in welchem bei der Obduktion eine Miliartuberkulose der Milz, der Lymphdrüsen und der Leber gefunden wurde, ohne dass man den Ausgangspunkt entdeckte.

Quincke (zitirt nach Jünger, s. u.) beschreibt einen Fall von myelogen-lymphatischer Leukämie, während dessen Verlauf eine Miliartuberkulose entstand. Zugleich mit der tuberkulösen Infektion sank die Zahl der Leukocyten, und die Milz- und Leberschwellung wurde geringer.

Stintzing (Tageblatt der Naturforscher und Aerzte in Heidelberg 1889) beschrieb einen Fall von lymphatischer Leukämie mit chronischer Tuberkulose. Während letztere Fortschritte machte und Kavernen in den Lungen nachweisbar wurden, besserte sich die Leukämie, indem die Lymphdrüsenschwellungen kleiner wurden und die Leukocytenzahl abnahm.

Auch in einem Falle Lichtheim's (Deutsche medizinische Wochenschrift, 30. September 1897, Vereinsbeilage) von Combination lymphatischer Leukämie mit Tuberkulose nahm die Leukocytenzahl mit dem Fortschreiten der Tuberkulose ab, indem sie von 250000 auf 8900 sank.

B aldw in (American journal of the medical sciences 1899) theilt einen Fall von lymphatischer Leukämie mit Tuberkulose mit.

Elsner und Groat (American journal of the medical sciences 1901) beschreiben einen Fall von myelogener Leukämie mit Tuberkulose. Mit dem Manifestwerden der Tuberkulose sank die Gesammtzahl der Leukocyten. Prozentualiter stieg die Zahl der mehrkernigen neutrophilen Elemente und die der Lymphocyten, während die der Myelocyten sank; im Beginn der Beobachtungen wurden 320000 , am Schlusse derselben 121000 weisse Blutkörperchen gezählt.

In einem von Jünger (Virchow's A rchiv Bd. CLXII) mitgetheilten Fall wurde bei Beginn der Tuberkulose eine Zunahme der Leukocyten von 40000 auf 120000 , später eine Abnahme bis auf 68000 constatirt. Unter den weissen Blutkörperchen befanden sich zahlreiche Markzellen, zum Theil mit Mitosen, grosse und kleine Lymphocyten, relativ spärlich polymorph- und vielkernige Elemente, sehr spärlich eosinophile Zellen. Zwei Tage vor dem Tode war eine Vermehrung der Leukocyten nicht mehr nachweisbar. Die anatomische Diagnose des Falles lautete: Miliare Tuberkulose besonders des Peritoneums, hyperplastische Lymphdrüsen mit Tuberkulose. Die Milz war vergrössert und wog $250 \mathrm{~g}$, das Knochenmark war tief dunkelroth und von käsigen Heerden durchsetzt.

Auf Grund dieser Beobachtungen lässt sich folgendes über die Combination der Leukämie mit der Tuberkulose sagen: Entweder entsteht im Verlaufe einer Leukämie eine Tuberkulose, deren Eingangspforte bisweilen nicht nachweisbar ist, oder die latente Tuberkulose eines Individuums wird im Verlaufe der Leukämie zu erneutem Aufflackern gebracht.

Auch in unserm Falle hatte die Tuberkulose einen Einfluss auf das Verhalten der Leukocyten. Während die Zahl derselben im Beginne der Beobachtung 426000 betrug, wurden kurz vor dem Exitus 283300 gezählt.

Am 3. März 1899, demjenigen Tage, an welchem zum ersten Male ein profuser Katarrh der Lunge festgestellt wurde und an welchem höchst wahrscheinlich die Propagation der Tuberkulose begann, waren nur 110900 Leukocyten vorhanden. So gross wie in einigen anderen Fällen der Litteratur war also in unserem der Einfluss der Tuberkulose auf die Leukocytenzahl nicht. Im übrigen war das Resultat der mikroskopischen Untersuchung der Organe folgendes:

Die Hyperplasie der Lymphdrüsen beruhte nicht lediglich auf einer Vermehrung der Lymphocyten, sondern man sah auch in der Pnlpa zahlreiche neutrophile und eosinophile Leukocyten und Riesenzellen vom Typus der des Knochenmarks (myeloide Umwandlung). Dass in den Lymphdrüsen mikroskopische käsige Heerde verstreut waren, ist bereits erwähnt.

Das Knochenmark bestand ganz aus Leukocyten, und namentlich in den weissen Theilen wurden rothe Blutkörperchen fast ganz vermisst. Man sah auf Schnitten wie in Abstrichpräparaten alle Leukocytenformen in regelloser Weise nebeneinander. Eeosinophile und Mastzellen waren sehr zahlreich; im grossen und ganzen waren hier dieselben Elemente wie im Blute vorhanden. Fett fehlte fast gänzlich.

In der Milz war die Pulpa ebenso wie in den Lymphdrüsen theilweise in myeloider Umwandlung begriffen, ausser mehr- und einkernigen neutrophilen und eosinophilen Zellen sowie Riesenzellen sah man hier auch noch viele Normoblasten. Auch die Milz enthielt viele kleine verkäste Heerde.

Der Ohrtumor, der etwa kleinbohnengross und von festweicher Consistenz war, sah auf dem Durchschnitt weiss aus, etwa wie eine Lymphdrüse. Einzelne rothe Blutgefässe durchzogen den Querschnitt. Mikroskopisch konnte festgestellt werden, dass die Grundsubstanz der Geschwulst aus fibrillärem Bindegewebe bestand, das von zahlreichen, meist stark und zum Theil sogar kollosal erweiterten Gefässen durchsetzt war, die alle Blut enthielten. Da letzteres fast ganz aus weissen Blutkörperchen bestand, konnte mit blossem Auge der Blutreichthum der Geschwulst nicht erkannt werden. Das interstitielle Gewebe war durchweg von Leukocyten durchsetzt, und zwar von ein- und mehrkernigen neutrophilen und eosinophilen Elementen; Mastzellen wurden nicht gesehen. Stellenweise waren ganz dichte Leukocytenanhäufungen zu sehen.

Es ist wohl zweifellos, dass es sich hier um eine leukämische Metastase, ein Myelom, handelte. Die Geschwulst muss ihrer sonstigen Struktur nach als Fibroangiom bezeichnet werden. Der Einwand, dass hier nur eine entzündliche Infiltration vorlag, ist aus dem Grunde nicht stichhaltig, weil die zahlreichen Gefässwände nirgends von Leukocyten infiltrirt erschienen. Auch das Rückenmark wurde mikroskopisch untersucht, es konnten aber keine Veränderungen gefunden werden.

Wir haben in diesen beiden Fällen und einem dritten hier nicht ausführlicher mitgetheilten die Angaben Löwit's über den Befund von Protozoën im leukämischen Blute nachgeprüft. Genau nach seinen Angaben haben wir Deckglastrockenpräparate $11 / 2$ bis 2 Stunden bei einer Temperatur von $110-120^{\circ} \mathrm{C}$ erhitzt, dann $1 / 2$ Stunde lang in einer Mischung von 1 Theil gesättigter wässeriger Thioninlösung und 2 Theilen Loeffler'schen Methylenblau gefärbt, in Wasser abgespült und dann mit Jodjodkalilösung $1: 2: 300$ bis 20 Sekunden differenzirt.

In der That konnten wir das Vorkommen von Gebilden, die den von Löwit als Parasiten beschriebenen ähnlich sind, constatiren; nur haben wir Geissel-, Sporulations- und Navikelformen niemals gesehen; auch gelang es uns nicht, in den fraglichen Elementen einen olivgrünen Farbenton hervorzurufen, sondern dieselben erschienen mehr blaugrün bis schwarzgrün. Vielleicht lag das daran, dass wir nur Merck'sches Thionin, nicht Thalheim'sches, welches Löwit benutzte, zur Verfügung hatten. Wir sahen parasitenähnliche Gebilde erstens in den grossen mononukleären Zellen, in denen sie auch Löwit beschreibt; seltener bemerkten wir sie in kleinen Lymphocyten, wo sie nach Löwit besonders häufig angetroffen werden sollen. Ferner sahen wir sie in polymorphkernigen Zellen, die aber kleiner waren als die gewöhnlichen neutrophilen Elemente. In diesen Zellen waren sie stets in grosser Menge vorhanden und oft von ganz beträchtlicher Grösse. Alle Uebergangsformen von grossen Kugeln zu elliptischen, ovalen und sichelförmigen Gebilden waren hier vorhanden.

Wir halten aber diese Elemente nicht für parasitärer Natur. Die in den grossen mononukleären Zellen sichtbaren Gebilde halten wir zum Theil für Farbstoffniederschläge, zum Theil aber für Degenerationsprodukte des Protoplasmas oder des Kernes. Die grösseren Gebilde in den kleinen mehr- und in vielen kleinen einkernigen Zellen sind aber unserer Ansicht nach Mastzellengranula, die durch die angewandte Methode deformirt worden sind.

Wir Inüssen uns in dieser Beziehung ganz an das anschliessen, 
was Türck und Litten und Michaelis über diesen Gegenstand gesagt haben. Interessant ist auch, dass in unseren Fällen die Zahl dieser sogenannten Parasiten der der Mastzellen stets parallel ging und dass dieselben deshalb im Fall 1, der nur sehr wenig Mastzellen hatte, stets nur in geringer Anzahl gefunden wurden.

In den Blutbildungsorganen unseres zur Obduktion gekommenen Patienten fanden wir überhaupt keine den Löwit'schen Parasiten ähnliche Elemente, auch nicht die von Löwit mit dem Namen "grüne Körper“ bezeichneten Gebilde.

Wir haben auch mit den Organen unseres verstorbenen $\mathrm{Pa}-$ tienten die Infektionsversuche Löwit's nachgeprüft, indem wir 12 Kaninchen mit dem genau nach Löwit's Vorschrift hergestellten Organsaft aus Milz, Lymphdrüsen und Knochenmark spritzten.

Drei unserer Versuchsthiere starben an Embolieen bald nach der Injektiou, vier im Verlaufe einiger Tage an Sepsis. Im Blute der letzteren wurde wohl eine Hyperleukocytose nachgewiesen, aber weder leukïmische Veräıderungen, noch Parasiten gefunden. Die übrigen fünf Thiere blieben am Leben und zeigten anfänglich eine geringgradige Hyperleukocytose, aber weder Parasiten, noch leukämische Veränderungen. Sämmtliche Thiere wurden getödtet, aber auch in den Organen fanden wir keine leukämischen Veränderungen. 